\title{
Multi-Dimensional Creativity: A Computational Perspective
}

This paper presents a multi-dimensional perspective for the study of creativity and formulates a framework for computational creativity that consists of 1) Culture; 2) Society; 3) Groups; 4) Individual, and 5) Brain. This framework enables the definition of functional relationships among these scales, and captures the effects of time within each scale. Its relevance and usefulness are shown firstly by classifying recent studies of computational creativity, and secondly by illustrating multi-dimensional approaches to the computational study of creativity with sample scenarios grown in a simulation system. The paper closes offering modelling guidelines for the computational studies of creativity.

Keywords: computational creativity, multi-agent simulation, cellular automata Subject classification codes: include these here if the journal requires them

\section{Introduction}

The scientific study of creativity spans across multiple areas of knowledge, however most research is conducted in disciplinary isolation. The ways in which scholars frame research questions related to creativity largely depend on their affiliations, i.e., the methods they trust, the variables and indicators accepted in their fields to develop and test hypotheses, their scope, definitions and assumptions about a complex concept such as creativity, and the ways causal arguments are built. The scientific study of creativity is not only divided by “cultures” (Mahoney and Goertz 2006), but also by the many levels at which creativity is analysed. Research programs today include the study of “creative brains” (Fink et al. 2010), “creative cognition” (Smith et al. 2006), “creative personality” (Martinsen 2011), “creative teams” (Gloor et al. 2012), “creative leadership” and “creative organizations” (Mathisen et al. 2012), “creative occupations” (Lee and Drever 2012), “creative products” (Besemer 1998; Haller et al. 2011), “creative cities” (Evans 2009), “creative industries” (Hotho and Champion 2012), 
“creative countries” (Kim 2007), and “creative eras” (Marshall 2009) -to name just a few.

Rather than pursuing an encompassing definition, in this paper creativeness across scales and domains is viewed as a relative value ascribed to persons, processes, products, populations, etc. In other words, a person, process, product, occupation, city, or industry is considered creative inasmuch as they exhibit properties that set them apart from their counterparts. Such properties are defined and accepted by a community and customarily include novelty, utility, and surprise or non-obviousness. The ascription of creative value ranges from routine to disruptive (Gero 1990; Kaufman and Beghetto 2009). Since the creation and evaluation processes occur at different scales and times, this paper regards creativity as a multi-level construct. A framework is presented here to support the study of creativity from multiple scale and time dimensions. Although this framework can guide all research work, we demonstrate its relevance in computational creativity, due to the methodological flexibility that computational models offer to define, inspect and scrutinize interactions across scale and time levels.

Computational creativity is a relatively recent and promising research approach that complements the current methods and techniques to understand and support creativity. Its origins in Artificial Intelligence (AI) help explain its emphasis so far on the modelling of individual processes, and the use of external (human) evaluations of performance and outcomes. In the traditional AI paradigm, the system architecture consists of autonomous agents interacting with an external environment and producing solutions that are assessed by human judges in terms of their quality and similarity to human solutions (Russell and Norvig 2005). Alternative ways of applying computational methods to the study of creativity from multiple dimensions are developed in this paper. 
A multi-dimensional perspective for the study of creativity is presented here. The aims of this work include: to enable new ways of thinking about creativity from different disciplines supported by computational models, to support communication between research traditions, and to map the units of analysis, variables and interactions across scales and time. The paper is organized as follows: Section 2 introduces key concepts and draws from the theoretical bases of this approach; Section 3 presents our framework and explains its main structural and functional aspects. Section 4 applies this framework to classify recent studies of computational creativity and illustrates multidimensional modelling approaches in a simulation system used to inspect fundamental questions about creativity and innovation. Section 5 closes the paper presenting modelling guidelines and implications for connecting computational methods to other research methods for the study of creativity.

\section{Background}

Integrating scientific disciplines is an intellectual effort that goes back to Comte’s hierarchy of sciences according to the scale and complexity of theoretical tools (Mayer and Lang 2011). The role of cultural mediation in the development of cognitive functions has its origins in the tradition of cultural psychology since Vygotsky (Moran and John-Steiner 2003). Research on the social-psychology of creativity began to illustrate the interaction between individual and external factors (Hennessey 2003). More recently, cultural-psychology creativity has extended that approach by shifting the architecture from a view of individual behavior “conditioned” by social factors and towards a more integrated view where interdependent relationships co-constitute complex creative systems (Glăveanu 2010). Ecological models of creative problem solving integrate cognitive, personality, and situational factors (Isaksen et al. 1993). Conceptual models where creativity is viewed as a social construct have been discussed 
(Simonton 2004; Westmeyer 2009), but they remain largely unconnected from empirical, experimental or computational studies.

Multilevel models that capture the interactions between psychological, social and cultural factors enable two complementary research directions. On the one hand, holistic explanations are possible by going up in the hierarchy drawing upon higher levels that moderate lower effects. On the other hand, reductionist explanations go down in the hierarchy to inspect lower-level factors that account for high-level phenomena (Koestler and Smythies 1969). For example, accounting for cultural constructs can be essential to understand individual attitudes to altruism (Sheldon et al. 2011). Likewise, individual cognitive styles have been studied to help explain and manage group conflict (Kim et al. 2012).

Contemporary personality research provides empirical support for the irreducibility postulate: i.e., "no scientific discipline is likely to subsume the others, all are needed” (Sheldon et al 2011). In the field of personality and well-being, multilevel approaches are recommended to study the complex interactions and effects among factors within and between levels of organization: cultural, social, personality, cognition and neural (van Mierlo et al 2005). Such integrated and interdisciplinary models should account for moderator relationships between levels of organization.

The Multilevel Personality in Context (MPIC) (Sheldon et al. 2011), The Five Fundamental Principles for an Integrative Science of Personality (McAdams and Pals 2006), and the Cognitive-Affect Personality System (CAPS) (Mischel and Shoda 1995) show how multiple levels of analysis can be integrated for a more reliable and complete understanding of complex human behavior -such as creativity. The MPIC model specifies the following levels: Culture, Social relations, and four levels of Personality: Self-Narratives, Goals/Motives, Traits/Dispositions, and Needs/Universals (Sheldon et 
al. 2011). Reviewers of the MPIC model further suggest the addition of situations to account for contextual factors beyond the bio-psychosocial (Mayer and Lang 2011). McAdams and Pals (2006) view personality as shaped by individuality, dispositional traits, characteristic adaptations, and life stories situated in a cultural environment that provides rules for the expression of trait tendencies, influences the content and timing of characteristic adaptations, and provides the canonical narrative forms out of which people make meaning of their lives.

In the study of creativity, Indurkhya (2012) addresses the interaction between system levels by asking where is the creativity when non-conscious or unintentional processes generate artifacts deemed as creative by an audience (i.e., works of art by a person diagnosed with schizophrenia or in cases of unexpected commercial success). A similar point can be made when considering the attribution of creativity to designs by Nature (McGrew 2012). Understanding the interplay between generative and evaluative processes of creativity has the potential to transcend such apparent paradox where at a given level it may seem like "there is nothing distinctive [...] that we can label as creative” (Indurkhya 2012). Paraphrasing Weisberg (1993), a multi-dimensional approach to creativity may reinforce the view that "creative works, even the most radical, are the result of [the coupling between] ordinary thinking [generative and evaluative] processes.”

In view of the disciplinary divides between fields where creativity is studied (psychology, anthropology, sociology, cultural geography, innovation research, design research, economics, linguistics, neuroscience), the study of such complex phenomenon requires cross-disciplinary perspectives that explore complementary levels of causality (Sternberg and Grigorenko 2001). In contrast to other research methods, computational creativity supports such cross-disciplinary reasoning (Meyer et al. 2009). 
Computational creativity refers to the scientific study of computational processes related to human creativity (Cardoso et al. 2009). This type of research in artificial creativity has been heavily dominated by generative systems focused on modelling the cognitive level, exploring the extents to which computers can produce solutions deemed as creative by unsuspecting judges. Paulus et al. (2010) present a generative system that combines individual and small-group creative processes, drawing from and transforming a corpus that is internal to the system, i.e., repositories of baseline solutions inspired by external (human) cultural references but with no goal to transform them.

Automated creativity further illustrates the gap between computational representations and human interpretations, such as the gap between written recipes and the imagined dish by cooks (Morris et al. 2012). This constitutes one of the main challenges of this field: that 'creative computers' ought to impact the external domain (poetry, music composition, painting, etc.) taking as inputs existing exemplary works to produce outputs that are evaluated by expert judges with a more complete access to the domain. For instance, in the case of written recipes, judges can easily imagine how a dish may taste (Morris et al. 2012). From a more pragmatic approach, in the field of Computer-Aided Innovation (CAI), computational tools are created and studied to assist designers in the creative process (Leon 2009).

Maher (2012) frames the need for evaluation criteria that are independent of the generative process. Jordanus (2011) suggests a standardized approach to evaluation where key components are identified, clear metrics are defined and tests are implemented. The work presented in this paper extends previous work ( ) and puts forward a structural and functional framework for the integrated study of 
computational creativity spanning system dimensions and therefore integrating views of creativity across disciplines.

\section{Multi-Dimensional Creativity}

The Multi-Dimensional Creativity (MDC) framework builds on the Idea-Agent-Society (IAS) triad of creative systems: epistemological, individual and social (Sosa et al. 2009). IAS synthesizes constructs from five influential theories, i.e.: exemplars, proponents, and communities (Ludwik Fleck); innovations, entrepreneurs and markets (Joseph Schumpeter); noosphere, strong spirit and culture (Edgar Morin); domain, individual and field (Mihalyi Csikszentmihalyi); and logic, genius and zeitgeist (Dean Simonton). The IAS framework maps out intrinsic processes within epistemic, human and social spheres (idea, agent and society, respectively), and six directed interaction processes between these spheres (Csikszentmihalyi 1988; Fleck 1981; Morin 1992).

MDC goes beyond the mapping of systemic dimensions and enables the definition of temporal and functional relationships in five scales of analysis: 1) Culture; 2) Society; 3) Group; 4) Individual; and 5) Brain. These relationships can be defined in computational studies as independent or interdependent, i.e., the former represent processes that occur only within a single scale in isolation, whilst the latter represent processes that are connected between scales. For example, a range of cognitive functions can be studied in a computational system, some of which can be assumed to emerge from explicit lower-level neural processes, others that are defined only within the cognitive level, and a third type that leads to higher-level personality or group processes. Figure 1 shows the MDC framework in a radial arrangement to explicitly avoid the implication of concentric circles where higher-level factors consist of aggregated units from lower levels (i.e., a group is not seen simply as a collection of individuals, etc.). 
[Insert Figure 1 here]

Dimension MDC-1, Culture, refers to the macro epistemological scale of creativity and addresses questions such as "How do systems of beliefs, language or taste change over time?”, “How may intellectual property law affect creativity and innovation in the short and long run?”, or “To what extent do the physical environment and communication practices determine the levels of creativity in an organization?”. Computational models at this scale can seek to grow these processes artificially by manipulating a range of initial factors and conditions, or they may consist of artificial systems that draw from or are inspired by real-world knowledge bases and corpora. MDC-1 relevant studies target topics such as: culture and technology evolution, organizational culture, language and semiotics, economic impacts, taste and traditions, public policy, mass media, intellectual property, cross-cultural comparisons, and creative environments.

Dimension MDC-2, Society, refers to the macro scale of human agency. It captures processes that account for the influence of -or seek to grow effects ondemographics, networks, migration, social influence and authority, roles and occupations, class structure, social capital, crowdsourcing, market segmentation, reputation and popularity, ethnic diversity, gender and aging, diffusion of innovations, and crowd behavior. Computational social models may instantiate the societal group as a separate class of variables with group-level behaviors, although the more common approach has been to view artificial societies as aggregate groups of autonomous agents which lack any explicit representation of their shared membership.

Dimension MDC-3, Group, refers to creativity phenomena that occurs at the scale of small to large groups of humans. This includes ideation sessions, team dynamics, communities of practice, family and peer support, co-creation, artist 
collectives, art commission, brainstorming, change management and leadership, deliberation, collaboration/competition strategies, workplace practices, groupthink, game theory, adopter categories. In computational creativity the difference between the social and group levels may be one of scale with the ensuing implementation challenges, and the option to have richer models of individual behavior in smaller groups. Modelling small groups may also facilitate the study of group-to-group interactions as well as individual awareness of group characteristics.

Dimension MDC-4, Individual, is the most common scale of study of creativity spanning cognitive science and psychology research. Beyond ‘creative cognition’ (intuition, insight, incubation, problem framing and solving, concept formation, representation, fixation, association, analogy, divergent thinking, abductive reasoning), processes on this scale also include personality types, motivation, curiosity, extroversion, mental health, addictions, emotions, risk aversion, well-being and lifestyle, habit, expertise, perception, biases, heuristics, etc. This is also the dominant approach in computational creativity where the main aim has been to build generative systems that typically produce a large number of solutions from which the researcher arbitrarily selects a few to either present as evidence of creativity or to present to a panel of judges (audiences or experts), who compare and evaluate the outputs.

Finally, dimension MDC-5, Brain, includes all creativity processes at the neural scale including neuroanatomy (brain asymmetry, neural circuits), neuromodulation (risk, arousal, novelty), brain stimulation, as well as neural network (NN) models of creative reasoning.

[Insert Table 1 here]

The MDC framework presented here accounts for multiple scales of creativity, each of which has been traditionally addressed in isolation as shown by the existing 
research programs in Table 1. MDC links these scales together and enables researchers across disciplines to explore top-down and bottom-up connections between these scales, as well as to distinguish time-based patterns within each scale.

\subsection{Time in MDC}

The MDC framework includes time factors across scales. Time is considered by the length and stability of the interactions, ranging from a few seconds as in studies of creativity and brain activity to millennia as in studies of creativity in human evolution. Therefore, the time scale is defined independently for each dimension. In MDC-1, culture, short-term processes of interest range from rapid changes over days or weeks such as those observed in the fashion industry (Mora 2006) and transient fads (Krapivsky et al. 2010). Mid-term processes of MDC-1 span over years and include geographical analyses of innovation and entrepreneurship (Lee et al. 2004), influence of knowledge and beliefs on creativity (Bhawuk 2003; Kim 2007), and the cultural assessment of creative ideas (Hempel and Sue-Chan 2010). Long-term processes that span from years to centuries in MDC-1 include archeological studies of the origins of human innovation and creativity (Elias 2012), and historical studies of culture and creativity (Marshall 2009; Tan 1997; Kuhn 2012).

In MDC-2, society, the shortest timescale includes events such as creativity in online collaboration and unconventional self-coordinated rapid responses to contingencies (Webb and Chevreau 2006; Crespo et al. 2007). Social processes that span weeks to months in their planning and execution include: word-of-mouth communication, and innovative bottom-up structures such as social movements in politics, sports, and festivals (Godes and Mayzlin 2004; Shepard 2011). More stable social relationships relevant to creativity grow over years and can extend through centuries and beyond, such as schools, awards and creative guilds; studies of population 
genetics, human behavioral ecology and evolutionary psychology also illustrate longterm social phenomena related to creativity (Mahmood 2008; Kijkuit and Van Den Ende 2007; Scott 2006; Becker 1997; Sunstein 2003).

In MDC-3, group, ephemeral interactions occur in periods of a few minutes or hours such as ideation sessions, conversation analysis and improvisation (Paulus and Nijstad 2003; Sawyer 2006; Björkman 2004; Lemons 2005). More stable events include project teamwork and conflict, workplace dynamics, creativity and leadership, creative performance over time, and classroom creativity (Chen 2006; Nemeth et al. 2004; James et al. 2004; Shalley and Gilson 2004; Tierney and Farmer 2011; Saracho 2012; Starko 2009). Long-term structures in MDC-3 may span for decades and longer, such as in the role of social networks and career development (Jones 2010; Ohly et al. 2010).

In MDC-4, individual, the research methods tend to focus on laboratory settings where particular cognitive mechanisms are studied over a few minutes, and may extend to include effects that last days or weeks, i.e., periods of incubation and a-ha moments of insight (Smith et al. 1995; Gero 2011; Storm and Angello 2010; Hennessey 2003; Gilhooly et al. 2012). Personal traits and processes that span over several years include formal education, ageing, childhood and mental health (Simonton 2004; Vygotsky 1990; Duffy 2006; Basu et al. 2011; Zhang and Niu 2013; Noori et al. 2012). Lastly, MDC-4 life-long phenomena such as giftedness, career trajectories and biographies represent the long-term dimension in this category (Sak 2004; Gardner 2011; Syed 2010).

Lastly, in MDC-5 brain activity is analyzed over short periods from seconds to minutes (Aziz-Zadeh et al. 2013, Kowatari et al. 2009; Green et al. 2012; Dietrich 2004; Dietrich and Kanso 2010). More persistent processes include the sustained effects of brain magnetic stimulation, drugs, sleep and dementia, as well as brain plasticity 
(Snyder et al. 2012; Fink et al. 2010; Rosenthal and Westreich 2010; Maquet and Ruby 2004; Miller and Hou 2004; Otte 2001). Long-term brain phenomena include the evolution of the human brain, the relation between brain development and language, and the neurobiology of nonhuman animal creativity (DeFelipe 2011; Christiansen and Chater 2008; Kaufman et al. 2011).

\subsection{Modeling}

MDC is a conceptual framework that aims to guide computational studies of creativity, although it is applicable to other approaches combining existing research methods. Cross-scale and time-based interactions open up a triple set of opportunities for the in silico or computational study of creativity, such as the following examples:

- MDC supports the framing of reductionist models, i.e., what neural mechanisms help explain team ideation processes such as productivity loss ( $A$ arrow in Figure 2); how group dynamics, societal and cultural norms shape the role of change agents or determine self-perception of creativity ( $B$ arrow in Figure 2$)$.

- $\quad$ MDC supports holistic studies across scales, i.e., how may cultural or generational attitudes to change explain and be explained by individual traits, group dynamics such as family, and social rituals ( $C$ arrow in Figure 2$)$; the twoway interaction between brain and language evolution ( $D$ arrow in Figure 2).

- $\quad$ MDC enables the framing of longitudinal studies, i.e., how sudden events may lead to long-term cultural changes, workplace policies or social movements ( $E$ arrow in Figure 2); how a short-lived experience may affect an individual's creative career, and the lives of their peers and pupils ( $F$ arrow in Figure 2).

The MDC framework accommodates various research traditions, approaches and units of analysis. Figure 2 depicts the MDC framework with scales on the vertical axis 
and time on the horizontal axis. Arrows show examples of the type of cross-scale and time-based interactions that MDC supports.

[Insert Figure 2 here]

\subsection{Evaluation}

In this section the MDC framework is applied to recent computational creativity studies with the aim to evaluate whether cross-level interactions are currently captured in the literature. All 34 full papers published in the proceedings of an international conference were selected for this exercise, including full papers and position papers (Maher et al. 2012). These were classified in one or more of the MDC scales according to their research aims and claims as stated by the author(s), as well as the target research agendas in position papers. Table 2 presents the 34 papers (rows) and their relation to the MDC scales (columns) - only the first author's surname is used for clarity.

[Insert Table 2 here]

As may be expected, a vast majority of papers (more than 90\%) in computational creativity address the individual scale MDC-4, mainly by describing generative systems that produce creative outputs which are selected by the researchers and, in some cases, evaluated by external judges. This widespread focus on the individual scale is explained by the origins of this field in Artificial Intelligence, but also by the "lone genius" myth of creative practice, the dominance of individual approaches to the study of creativity in cognitive, personality and biographical fields, and the reductionist belief that other scales will ultimately build upon the individual dimension once it's well understood (Johnson 2012).

The most common type of paper overall (41\%) reports corpus-based generative systems, i.e., where the researcher selects a set of exemplars to use them as the basis for the synthesis of new solutions. The range of themes and domains is varied: poetry 
generators that use newspaper articles as input, music generators from sample classical music and from non-musical audio signals, poster generators that modify existing designs, and recipe generators based on recipes gathered from specialized websites.

These archetypical papers can also be seen as addressing MDC-1 not because they aim to model cultural changes within the system, but because they take repositories of human culture as inputs to produce their output. Such papers account for $67 \%$ of papers dealing with the MDC-1 scale, the rest being mainly position papers that underline the importance of including the cultural dimension in computational studies. The two exceptions are Baydin et al. (2012) whose algorithm is based on the concept of “memes” or units of culture and Gabora and DiPaola (2012) who specifically aim to model cultural evolution.

Societal factors (MDC-2 scale) are mentioned in 20\% of all papers, half of these by reporting evaluation by audiences or panels of experts. The four cases that explicitly refer to the modelling of social processes are all position papers proposing approaches and analyzing the potential benefits of accounting for the social scale of creativity in computational systems. Group creativity (MDC-3) is targeted in less than 10\% of all cases, two position papers and one research paper reporting results from a computational study of group influence (Sosa and Gero 2012).

Only two papers (6\% of all entries) refer to the brain scale (MDC-5), in both cases by using neural network approaches for the implementation of generative systems (Gabora and DiPaola 2012, Hoover et al. 2012). Elsewhere, progress is being made explicitly modelling creative neural processes (Iyer et al. 2009).

The following observations can be made from this MDC mapping exercise: a) the field of computational creativity is characterized by studies that focus on individual generative processes (MDC-4); b) most of the generative systems reported take human 
cultural corpora as inputs, training sets or exemplar cases; and c) less than 1 in 4 papers span more than two MDC scales -half of these being position papers suggesting future research directions. The MDC framework provides a modelling structure to support the ongoing development of the field towards tackling multi-level research questions.

More specifically, this mapping exercise of the literature using the MDC scales, supports the framing of possible modelling approaches. Here we develop a few possible research scenarios for illustration purposes:

- Computational models that integrate group and social evaluation of creativity explicitly within the system, i.e., "automated critics” or “artificial audiences” capable of simulating the assessment criteria and patterns of human judges. Such evaluations could account for multiple decisive conditions such as agreement/disagreement, public opinion, expert endorsement, and different scales and levels of domain expertise. A sample research question for such systems is "How may a computational system automatically distinguish innovative from ordinary designs in product catalogues from different industries?”

- Computational models of neuro-mechanisms related to the synthesis as well as to the evaluation of creativity. Such systems could capture the connections between neural mechanics and other scales, particularly cognitive and group processes. A relevant research question is "How may basic functions such as word retrieval and short term memory moderate the generation and evaluation of creative ideas in brainstorming?”

- Computational models of personality and motivation in the synthesis as well as the evaluation of creativity, for example systems that create or evaluate artifacts based on emotional predispositions, gender and age differences, and other 
personality dimensions. In such models, creative behavior can be analyzed as moderated by environmental cues. Relevant research questions include "How may personality traits such as extroversion and conformity moderate the drive to generate or the assessment of new ideas?”.

- Accounting for the effects of time across scales can lead to computational models that help understand the conditions that make an ephemeral event or a new idea become influential in modifying a culture or a domain of practice. By modelling managerial practices and informal interactions in teams and organizations, computational models can help grow scenarios where leadership creates and sustains cultures of innovation. Generative systems that integrate time-based factors could model perseverance, anticipation, habit, and the effects of expertise and mastery.

In summary, mapping recent computational creativity studies applying MDC scales is valuable because it allows to clarify assumptions and units of analysis, connect issues, identify gaps and formulate new proposals for the advancement of the field. MDC suggests ways to transcend the current focus on individual generative systems that draw from a hand-picked set of external sources to cleverly produce new solutions which may be judged as creative by external experts and audiences. As computational models integrate multi-scale factors, they can draw from and inform a multiplicity of ongoing research methods to support and complement alternative ways of understanding and studying creativity.

\subsection{Multi-scale MDC computational modelling}

An example of how MDC applies in computational creativity is offered in this 
subsection to illustrate the type of questions, target processes, experimental variables and conditions, and ultimately the type of outputs and expected contributions using cross-scale computational creativity in multidisciplinary research programs.

The model discussed here belongs to a class of computational simulations used to gain qualitative understanding of human and social behavior and it is also one of the simplest to code (Nowak and Lewenstein 1996). Montfort and Fedorova (2012) recommend such 'small-scale systems' in computational creativity as they are easily communicated, implemented, understood and modified. Only a few studies have used this modelling formalism to reason about creativity and innovation (Goldenberg and Efroni 2001; Adamatzky and Wuensche 2013). The model is a type of two-dimensional cellular automata where reactive agents move about and interact with each other in a shared environment guided by simple representations and behaviors. Agents in this model are viewed as having an opinion or idea encoded as a chain of numerical values. They communicate with neighboring agents to exchange and influence their ideas. Individual differences give each agent a unique profile on how it traverses the social space, the initial set of ideas assigned to them, and the strength of its ideas (their abilities to change opinions). During a simulation every agent has a location in a coordinate space expressed by a pair $(x, y)$ of integers, an individual idea expressed by a set $\left(k_{i} . . n\right)$ of size $n$ of integers, and individual traits that regulate their behavior also expressed by a set $\left(r_{i} \ldots m\right)$ of size $m$ of integers. Set $k$ contains the values to be exchanged by agents, while set $r$ contains individual parameters that determine how individual agents behave.

In the initial state of this dynamic system, agents are instantiated from a super class and stored in an array with pseudo-random values assigned on the three sets, following a distribution of choice. Figure 3 shows 20 agents at step 0 with a unique 
location in a torus square grid and a color combination representing their idea values. Simulations with millions of agents are feasible with current personal computers, but we follow the 'small-scale system' guideline to maximize clarity. At every step, agents decide where to move, who to contact and whether to exchange or not an idea with one or more of their neighbors. This decision is made by a combination of randomness (agents' location and choice of idea value to exchange) and individual characteristics (idea value and strength to influence other agents). Full details of this stochastic system are given elsewhere (Sosa and Gero 2012) where multiple combinations of these parameters have been studied.

[Insert Figure 3 here]

A set of typical results are mapped in Figure 4, where idea diversity is plotted for three population sizes: 100, 200 and 400 agents. Two measures are used for estimating idea diversity on every step: origIdeas and diffIdeas . The former refers to the number of ideas held by only one individual in the population, while the latter refers to the number of different ideas across the population. Another way to see these two diversity indicators is: diffIdeas tells us how diverse ideas are in the whole group, origIdeas tells us how many individuals in the group have a uniquely different idea (Sosa and Gero 2012).

Lastly, ideasBreadth refers to how many positions or variables are used to represent ideas and ideasDepth to how many values are possible for each variable. In other words, the former captures how many issues are being discussed in the group, while the latter captures how varied are each of those issues. Suppose that a group of people get together to discuss recent laws on gay marriage or recreational drugs, then both ideasBreadth and ideasDepth will be rather short since opinions are framed as yes/no in both cases, with perhaps few other circumstances considered. In contrast, in 
an ideation session where groups discuss future market strategies, scientific research proposals or budget allocations, ideasBreadth will be as long as the number of issues included (possibly fixed at initial time), and ideasDepth will be as long as the number of alternatives generated (certainly expanding during a simulation). The results shown in Figure 4 are with ideasBreadth $=8$, ideasDepth $=4$ over 1500 simulation steps; cases are average trends of 100 simulations runs varying the random seed generator.

Two aspects of Figure 4 are relevant in this context: firstly, regardless of the values assigned in this type of models (grid size, number of agents, number of neighbors, decision to move or to exchange values), the final outcome is always identical: total group convergence, i.e., origIdeas $=0$ and diffIdeas $=1$. Another way to say this is that in these simple models of agents that exchange ideas, the final outcome is always total consensus on one dominant idea. Axelrod (1997) presents models where a notion of compatibility is implemented, whereby agents only exchange ideas if one of their values is the same. This rule allows for 'regional convergence' where agents form clusters of agents who share an idea and who do not have contact with other incompatible agents. The second significant observation here is that not all convergence is the same: under certain parameters, the rate of convergence in a group may accelerate or slow down, for example as a result of increasing redundant interactions between agents who already agree on some or all their ideas.

[Insert Figure 4 here]

Group convergence is important when reasoning about creativity because novel ideas are considered creative when a community agrees upon their novelty and usefulness (Kaufman and Beghetto 2009). However, for these systems to be relevant as reasoning aids in the study of creativity, they need to support divergence. In simulations with rich enough idea landscapes, a type of 'intrinsic divergence' is already observed in 
these convergent models: as agents exchange values, new combinations are generated (Sosa and Gero 2005). To that extent, the 'winning' or dominant idea in these systems is seldom or never (depending on the size of the idea space) one of the ideas originally assigned at initial simulation step, but a mutation collectively shaped by the exchange of ideas over time. A more relevant way to include divergence in these models is to include a threshold inspired by classic studies of the human bias to avoid monotonous, homogeneous stimuli (Berlyne 1970).

With such mechanism, as agents are repeatedly exposed to the same idea values, their probability of generating a new value increases. Figure 5a illustrates such case in a group of 20 agents where agent \#18 introduces a new value after recurrently encountering sameness with their neighbors. As expected, the chances of these nonconformist individuals to actually trigger a group change are slim, in most cases they are overcome by the convergent wave and adopt again the dominant idea due to the influence of neighbors. However, in some cases the new values do spread in a population, mostly in novel combinations of new and old ideas. Figure 5b shows the same group where the idea first introduced by agent \#18 has spread in three variants although the agent itself has by then reverted to the majority value.

\section{[Insert Figure 5 here]}

By introducing divergence in these models, they now support reasoning about change cycles. Figure 6 shows a group of 200 agents iteratively building consensus (diversity decreasing as in Figure 4) punctuated by 'spikes' of novelty over 20,000 simulation steps. These sudden increases of diversity are triggered by individuals changing one random idea value when diffIdeas $\leq 5 \%$. With a pool of new ideas introduced, diversity rapidly increases by combination with old ideas and this process 
continues until recurrent exchange slows down the generation of new ideas and gradually falls toward group convergence again.

[Insert Figure 6 here]

Varying the novelty threshold and the degree of novelty as well as other model parameters, the frequency and amplitude of cycles can be manipulated. In fact, there are numerous possible ways of growing such cycles of divergence and convergence even in these simple models. As argued earlier in this paper, the choices will largely depend on the intentions by the researchers and what their fields accept as valid assumptions and constructs. At this point, MDC offers clear options to locate sources and factors of change across dimensions. Even in such elementary systems, the alternatives are numerous.

MDC-1 Culture can guide the encoding of ideas, ranging from binary decisions as in yes/no referendums to topics that require increasingly longer coding schemas such as consensual group decisions or group ideation for open-ended problems. Knowledge representation can also be studied by including domain constraints, such as intrinsic relations of inclusion or exclusion between ideas or to account for re-structuring of knowledge schema. MDC-1 Culture can also address the shape and characteristics of the environment; while in cellular automata it is customary to represent this as a continual lattice in a two-dimensional space, topological features, such as social networks and idea sharing platforms remain to be studied.

MDC-2 Society can be used in these systems to define how agents traverse the space and cross boundary regions. This would help reasoning about practices and limits of movement as a way to understand the effects of migration in creativity and innovation (Hansen and Niedomysl 2009). Social structures of influence that can be included to study divergence and convergence include gatekeeping roles to endorse and 
promote new ideas, social norms of communication and exchange of ideas between agents, and social identity as a way to settle and form stable communities. Repeated success in triggering a group change could be used to build reputation levels in these systems where followers could build expectations around an elite group of agents.

MDC-3 Groups can help inform the definition of neighborhood type and size, principles of team formation with shared goals and emergence of leadership in small groups, etc. Turn-taking and other coordination strategies to influence the direction of change can be included in these systems, as well as biases and dilemmas of group collaboration informed by game theory (Wong 2012).

MDC-4 Individual and MDC-5 Brain can be used to account for differences between agents (openness to change, extroversion) including changes over time as a result of experience (conformity bias, boredom) and acquired abilities (learning, brain plasticity). Extending these models in more complete cognitive and neural models would move them closer to multi-agent architectures and possibly beyond the 'smallscale systems' principle adopted in this paper. One basic type of question in these two dimensions that can be explored in such models is: "How wide do individual differences need to be in order to generate extraordinary change events in the group?” (Weisberg 1993).

\section{Discussion}

Blunt (2010) states that “creativity is quintessentially a neurodevelopmental phenomenon”. Such views are pervasive across disciplines and traditions where creativity is studied: assumptions of what type of phenomenon is under scrutiny largely influence the questions, methods and claims. This paper has introduced a structured way of re-thinking creativity from a multi-level perspective. Multi-Dimensional Creativity (MDC) is relevant for all modes of inquiry, and has been discussed at length here in the 
context of computational creativity due to its methodological suitability to implement such studies.

MDC helps to frame the study of computational systems of agents, groups and societies that interact to generate and evaluate new ideas in reference to a shared domain that is internal to the system. Depending on the type and level of research question, cross-scale computational studies may establish pre-defined and uniform processes at some levels, while setting other processes as experimental at the same or different levels. For instance, if the researchers are interested in the possible ways in which neural activation determines how different individuals cope with failure (Davis et al. 2012), a system can be devised where a range of cultural, social, group and individual phenomena relevant to creativity is manipulated as control, while variations of the neural activity of individuals within certain social situations can be tweaked to capture possible causes and effects throughout the system. In such system, top-down changes can be introduced experimentally to inspect the transition levels at which social situations and neural activation in some individuals replicate target cases or rates of cultural change.

As with other types of inductive research, MDC modelling can be used to collect data systematically in an attempt to develop a theory or hypothesis. Inductive methods are valuable in new lines of enquiry where limited knowledge is insufficient to deduce testable propositions (Saunders et al. 2011). MDC models are seen here as weak computational creativity according to the AI nomenclature (Al-Rifaie and Bishop 2012). In distinction to strong positions that seek to address the conundrum "Can computers ever be creative?", weak positions explore the value of algorithmic studies of creativity in helping us to develop and empirically evaluate very specific and explicit ideas about this complex topic. As such, MDC models are not expected to provide strong evidence 
for new theories, or conclusive evidence to support or challenge current theoretical constructs. Their role is exploratory, their value is to aid reasoning, and they can be seen as an inductive approach to the study of creativity: MDC models help demonstrate what is possible, with the advantage of explicitly representing the mechanisms and dynamics at work.

In addition to multi-scale questions, possible longitudinal studies can be analyzed based on the MDC framework where time effects within and across scales can be systematically inspected. In an early study, questionnaires were applied to a large group of children in 1958 and again in 1980 showing that a significant relationship existed between having a mentor and creative achievement (Torrance 1981). Computational studies of mentorship can implement several rules of interaction between generative agents with a focus on hierarchies of expertise in order to dissect the principles and types of knowledge transfer between individuals to minimize learning curves or to challenge conventional practices. For instance, studies of the link between childhood and adulthood creativity have yielded contradictory outcomes: whilst Albert (1996) suggested that creativity was typically not maintained, Keegan (1996) found children's creativity to be a predictor of adult creativity. As with other factors related to creativity, answers are unlikely to be straightforward, and computational models can be enlightening to understand the type of life events that are more likely to nurture or suppress creativity through life stages (Casas 2003).

Based on the work presented in this paper, the following guidelines are provided to guide multi-dimensional computational modelling of creativity, building on the evaluation guidelines by Jordanus (2011).

- Guideline \#1: Scales to be included within the model 
o Define primary and complementary scales in the model; whilst empirical validation may not be possible across levels, computational explorations systematically support alternative thinking in scales of interest.

o Identify level variables (experimental and dependent) that represent target factors and observable behaviours or patterns of interest. Background literature from several disciplines are necessary to inform the formulation of contextual conditions.

o Define inputs and outputs at target levels, establishing the bootstrapping strategies of the model.

- Guideline \#2: Processes and links between scales

o Establish explicit connections above/below primary levels in the model.

o Define irreducible factors, causal links and whether the model is being used for holistic or reductionist purposes.

o Identify internal/exogenous factors to the system.

- Guideline \#3: Processes and links across time

o If relevant, establish time-based conditions, processes and variables of interest.

o Ensure that the targeted time series are reproducible to allow for experimental treatment.

- Guideline \#4: Define system outputs

o Define type and range of outputs, identifying extreme points such as non-creative to creative artifacts

o Capture and analyze aggregate data, model tuning and refinement

- Guideline \#5: Evaluation metrics 
o Validity may be achievable in some models where relevant empirical data exists at the primary level(s) of interest, but this may be inaccessible and even undesirable for exploratory models.

o Usefulness and relevance of such systems are ultimately defined by their aids as thinking tools, to explore hypotheses, to identify and connect issues across scales, to articulate conversations between disciplines.

\section{Acknowledgements}

This work was supported in part by the US National Science Foundation under grant number SBE-0915482. Any opinions, findings, and conclusions or recommendations expressed in this material are those of the authors and do not necessarily reflect the views of the National Science Foundation.

\section{References}

Adamatzky, A., \& Wuensche, A. (2013) On creativity and elementary cellular automata. Complex Systems, 22(4).

Adesope, O., Lavin, T., Thompson, T., and Ungerleider, C. 2010. A systematic review and meta-analysis of the cognitive correlates of bilingualism. Review of Educational Research 80(2):207-245.

Albert, R. (1996). Some reasons why childhood creativity often fails to make it past puberty into the real world. New Directions in Child Development, 72, 3-30.

Al-Rifaie, M.M. and Bishop J.M., (2012), Weak vs. strong computational creativity, computing, philosophy and the question of bio-machine hybrids. 5th AISB Symposium on Computing and Philosophy, University of Birmingham, UK.

Anderson, C., and Kilduff, G. J. 2009. Why do dominant personalities attain influence in face-to-face groups? The competence-signaling effects of trait dominance. Journal of Personality and Social Psychology, 96(2):491-503.

Axelrod, R. M. (1997). The Complexity of Cooperation: Agent-Based Models of Competition and Collaboration. Princeton University Press. 
Aziz-Zadeh, L., Liew, S. L., \& Dandekar, F. (2013). Exploring the neural correlates of visual creativity. Social Cognitive and Affective Neuroscience, 8(4), 475-480.

Bassett-Jones, N. 2005. The paradox of diversity management, creativity and innovation. Creativity and Innovation Management 14(2):169-175.

Basu, N., Nadeem, N. A., \& Wani, T. A. (2011). A comparative study of creative potential and study habits of academically gifted male and female students. European Journal of Education and Learning, 10, 14-19.

Baydin, A. G., De Mantaras, R. L., and Ontanon, S. (2012) Automated generation of cross-domain analogies via evolutionary computation, Third International Conference on Computational Creativity, 25-32.

Becker, H. S. (1997). Outsiders: Studies in the Sociology of Deviance. Simon and Schuster.

Berlyne, D. E. (1970). Novelty, complexity, and hedonic value. Perception \& Psychophysics, 8(5), 279-286.

Besemer, S. P. (1998). Creative product analysis matrix: testing the model structure and a comparison among products. Creativity Research Journal, 11(4), 333-346.

Bhawuk, D. P. (2003). Culture's influence on creativity: the case of Indian spirituality. International Journal of Intercultural Relations, 27(1), 1-22.

Björkman, H. (2004). Design dialogue groups as a source of innovation: factors behind group creativity. Creativity and Innovation Management, 13(2), 97-108.

Blunt, S. (2010). The creative brain: fundamental features, associated conditions and unifying neural mechanisms. Neurology of Music, 31.

Cardoso, A., Veale, T., \& Wiggins, G. A. (2009). Converging on the divergent: The history (and future) of the international joint workshops in computational creativity. AI Magazine, 30(3), 15.

Casas, A. K. (2003). Childhood Playfulness as a Predictor of Adult Playfulness and Creativity: A Longitudinal Study. PhD Dissertation, Virginia Polytechnic Institute.

Chen, M. H. (2006). Understanding the benefits and detriments of conflict on team creativity process. Creativity and Innovation Management, 15(1), 105-116.

Christiansen, M. H., \& Chater, N. (2008). Language as shaped by the brain. Behavioral and Brain Sciences, 31(05), 489-509. 
Crespo Cuaresma, J., Hlouskova, J., \& Obersteiner, M. (2007). Natural disasters as creative destruction? Evidence from developing countries. Economic Inquiry, 46(2), 214-226.

Csikszentmihalyi, M. (1988) Society, culture, and person: a systems view of creativity. In R.J. Sternberg (Ed.) The Nature of Creativity, Cambridge University Press, pp. 325-339.

Davis IV, H., van Anders, S. M., Ngan, E. T., Woodward, T. S., Van Snellenberg, J. X., Mayberg, H. S., \& Liotti, M. (2012). Neural, mood, and endocrine responses in elite athletes relative to successful and failed performance videos. Journal of Clinical Sport Psychology, 6(1), 6.

DeFelipe, J. (2011). The evolution of the brain, the human nature of cortical circuits, and intellectual creativity. Frontiers in Neuroanatomy, 5.

Dietrich, A. (2004). The cognitive neuroscience of creativity. Psychonomic Bulletin \& Review, 11(6), 1011-1026.

Dietrich, A., \& Kanso, R. (2010). A review of EEG, ERP, and neuroimaging studies of creativity and insight. Psychological bulletin, 136(5), 822.

Dollinger, S. J., Urban, K. K., and James, T. A. 2004. Creativity and openness: Further validation of two creative product measures. Creativity Research Journal 16(1):35-47.

Duffy, B. (2006). Supporting Creativity and Imagination in the Early Years. McGrawHill International.

Duflou, J. R., and Verhaegen, P. A. 2011. Systematic innovation through patent based product aspect analysis. CIRP Annals-Manufacturing Technology 60(1):203206.

Evans, G. (2009). Creative cities, creative spaces and urban policy. Urban studies, 46(56), 1003-1040.

Fink, A., Grabner, R. H., Gebauer, D., Reishofer, G., Koschutnig, K., \& Ebner, F. (2010). Enhancing creativity by means of cognitive stimulation: Evidence from an fMRI study. NeuroImage, 52(4), 1687-1695.

Finke, R. A., Ward, T. B., and Smith, S. M. 1992. Creative Cognition: Theory, Research, and Applications. Cambridge: MIT Press.

Fischer, G., Scharff, E., and Ye, Y. (2004). Fostering social creativity by increasing social capital. In Huysman, M. and Wulf, V. (Eds.) Social Capital and Information. Cambridge: MIT Press. 55-399. 
Fleck, L. (1981). Genesis and Development of a Scientific Fact. University of Chicago Press.

Florida, R. (2005). Cities and the Creative Class. Routledge.

Gabora, L. and Dipaola, S. (2012) How Did Humans Become So Creative? A Computational Approach. Third International Conference on Computational Creativity, 203-210.

Gardner, H. E. (2011). Creating minds: An anatomy of creativity as seen through the lives of Freud, Einstein, Picasso, Stravinsky, Eliot, Graham, and Ghandi. Basic Books.

Gero, J. S. (1990). Design prototypes: a knowledge representation schema for design, $A I$ Magazine 11(4):26-36.

Gero, J. S. (2011). Fixation and commitment while designing and its measurement. The Journal of Creative Behavior, 45(2), 108-115.

Gilhooly, K. J., Georgiou, G., \& Devery, U. (2013). Incubation and creativity: Do something different. Thinking \& Reasoning, 19(2), 137-149.

Glăveanu, V. P. 2010. Paradigms in the study of creativity: Introducing the perspective of cultural psychology. New Ideas in Psychology 28(1):79-93.

Gloor, P. A., Grippa, F., Putzke, J., Lassenius, C., Fuehres, H., Fischbach, K., \& Schoder, D. (2012). Measuring social capital in creative teams through sociometric sensors. International Journal of Organisational Design and Engineering, 2(4), 380-401.

Godes, D., \& Mayzlin, D. (2004). Using online conversations to study word-of-mouth communication. Marketing Science, 23(4), 545-560.

Goldenberg, J., \& Efroni, S. (2001). Using cellular automata modelling of the emergence of innovations. Technological Forecasting and Social Change, 68(3), 293-308.

Green, A. E., Kraemer, D. J., Fugelsang, J. A., Gray, J. R., \& Dunbar, K. N. (2012). Neural correlates of creativity in analogical reasoning. Learning, Memory, 38(2), 264-272.

Haller, C. S., Courvoisier, D. S., \& Cropley, D. H. (2011). Perhaps there is accounting for taste: Evaluating the creativity of products. Creativity Research Journal, 23(2), 99-109.

Hansen, H. K., \& Niedomysl, T. (2009). Migration of the creative class: evidence from Sweden. Journal of Economic Geography, 9(2), 191-206. 
Hempel, P. S., \& Sue-Chan, C. (2010). Culture and the assessment of creativity. Management and Organization Review, 6(3), 415-435.

Hennessey, B. A. 2003. Is the social psychology of creativity really social? Moving beyond a focus on the individual. In Paulus, P. B., and Nijstad, B. A. Group Creativity: Innovation through Collaboration. Oxford University Press. 181201.

Hoover, A.K., Szerlip, P.A., Norton, M.E., Brindle, T. A. (2012). Generating a complete multipart musical composition from a single monophonic melody with functional scaffolding. Third International Conference on Computational Creativity, 111-118.

Hotho, S., \& Champion, K. (2011). Small businesses in the new creative industries: innovation as a people management challenge. Management Decision, 49(1), 29-54.

Indurkhya, B. 2012. Whence is creativity? In International Conference on Computational Creativity, 62-66.

Isaksen, S. G., Puccio, G. J., and Treffinger, D. J. 1993. An ecological approach to creativity research: Profiling for creative problem solving. The Journal of Creative Behavior 27(3):149-170.

Iyer, L. R., Doboli, S., Minai, A. A., Brown, V. R., Levine, D. S., \& Paulus, P. B. (2009). Neural dynamics of idea generation and the effects of priming. Neural Networks, 22(5), 674-686.

James, K., Brodersen, M., \& Eisenberg, J. (2004). Workplace affect and workplace creativity: A review and preliminary model. Human Performance, 17(2), 169194.

Johnson, C. (2012). The creative computer as romantic hero? Third International Conference on Computational Creativity, 57-61.

Jones, C. (2010). Finding a place in history: Symbolic and social networks in creative careers and collective memory. Journal of Organizational Behavior, 31(5), 726748.

Jordanous, A. 2011. Evaluating evaluation: Assessing progress in computational creativity research. In Proceedings of the Second International Conference on Computational Creativity, 102-107. 
Jung, R. E., Segall, J. M., Jeremy Bockholt, H., Flores, R. A., Smith, S. M., Chavez, R. S., and Haier, R. J. 2010. Neuroanatomy of creativity. Human Brain Mapping 31(3): 398-409.

Kaplan, S., Brooks-Shesler, L., King, E. B., \& Zaccaro, S. (2009). Thinking inside the box: How conformity promotes creativity and innovation. E. A. Mannix, M. A. Neale, and J. A. Goncalo (Eds.) Creativity in Groups, Emerald, pp. 229-265.

Kaufman, A. B., Butt, A. E., Kaufman, J. C., \& Colbert-White, E. N. (2011). Towards a neurobiology of creativity in nonhuman animals. Journal of Comparative Psychology, 125(3), 255.

Kaufman, J. C., and Beghetto, R. A. 2009. Beyond big and little: The four c model of creativity. Review of General Psychology 13(1):1-12.

Keegan, R. (1996). Creativity from childhood to adulthood: A difference of degree and not of kind. New Directions in Child Development, 72, 3-30.

Kijkuit, B., \& Van Den Ende, J. (2007). The Organizational Life of an Idea: Integrating Social Network, Creativity and Decision-Making Perspectives. Journal of Management Studies, 44(6), 863-882.

Kim, K. H. (2007). Exploring the interactions between Asian culture (Confucianism) and creativity. The Journal of Creative Behavior, 41(1), 28-53.

Kim, M. J., Choi, J. N., \& Park, O. S. (2012). Intuitiveness and creativity in groups: Cross-level interactions between group conflict and individual cognitive styles. Social Behavior and Personality: an international journal, 40(9), 1419-1434.

Koestler, A., Smythies, J. R. (eds.) (1969). Beyond reductionism: new perspectives in the life sciences. Hutchinson, London.

Kowatari, Y., Lee, S. H., Yamamura, H., Nagamori, Y., Levy, P., Yamane, S., \& Yamamoto, M. (2009). Neural networks involved in artistic creativity. Human Brain Mapping, 30(5), 1678-1690.

Krapivsky, P. L., Redner, S., \& Volovik, D. (2011). Reinforcement-driven spread of innovations and fads. Journal of Statistical Mechanics: Theory and Experiment, 2011(12), P12003.

Kuhn, S. L. (2012). Emergent patterns of creativity and innovation in early technologies. In S. Elias (Ed.) Origins of Human Innovation and Creativity, 6988.

Lee, N., \& Drever, E. (2012). The Creative Industries, Creative Occupations and Innovation in London. European Planning Studies, (ahead-of-print), 1-21. 
Lee, S. Y., Florida, R., \& Acs, Z. (2004). Creativity and entrepreneurship: a regional analysis of new firm formation. Regional Studies, 38(8), 879-891.

Lemons, G. (2005). When the horse drinks: Enhancing everyday creativity using elements of improvisation. Creativity Research Journal, 17(1), 25-36.

Lessig, L. 2008. Remix: Making Art and Commerce Thrive in the Hybrid Economy. Penguin Press HC.

Leon, N. (2009). The future of computer-aided innovation. Computers in Industry, 60(8), 539-550.

Lubart, T. 2010. Cross-cultural perspectives on creativity. In Kaufman, J. C., and Sternberg, R. J., eds. 2010. The Cambridge Handbook of Creativity. Cambridge University Press. 265-276.

Maher, M.L. 2012. Computational and collective creativity: Who’s being creative?. In International Conference on Computational Creativity, 67-71.

Maher, M.L., Hammond, K., Pease, A., Perez y Perez, R., Ventura, D., and Wiggins, G. (Eds.) (2012). Third International Conference on Computational Creativity.

Mahmood, B. (2008). Sociological Study of Behavioral Change in Textile Manufacturing Organizations of Punjab. PhD Dissertation, University of Agriculture Faisalabad.

Mahoney, J., \& Goertz, G. (2006). A Tale of Two Cultures: Contrasting Quantitative and Qualitative Research. Political Analysis, 14(3), 227-249.

Maquet, P., \& Ruby, P. (2004). Insight and the sleep committee. Nature, 427(6972).

Marshall, P. (2009). Six Renaissance Men and Women: Innovation, Biography and Cultural Creativity in Tudor England, c. 1450-1560. The English Historical Review, 124(510), 1160-1162.

Martinsen, Ø. L. (2011). The creative personality: a synthesis and development of the Creative Person Profile. Creativity Research Journal, 23(3), 185-202.

Mathisen, G. E., Einarsen, S., \& Mykletun, R. (2012). Creative leaders promote creative organizations. International Journal of Manpower, 33(4), 367-382.

Mayer, J. D., \& Lang, J. L. (2011). A Three-Dimensional View of Personality. Psychological Inquiry, 22(1), 36-39.

McAdams, D. P., \& Pals, J. L. (2006). A New Big Five: Fundamental Principles for an Integrative Science of Personality. American Psychologist, 61(3), 204-217.

McCoy, J. M., and Evans, G. W. 2002. The potential role of the physical environment in fostering creativity. Creativity Research Journal 14(3-4):409-426. 
McGrew, S. 2012. Creativity in Nature. In Swan, L;, Gordon, R., and Seckbach, J., eds., Origin(s) of Design in Nature. Springer Netherlands. 43-55.

Meyer, M., Lorscheid, I., \& Troitzsch, K. G. (2009). The development of social simulation as reflected in the first ten years of JASSS: a citation and co-citation analysis. Journal of Artificial Societies and Social Simulation, 12(4), 12.

Miller, B. L., \& Hou, C. E. (2004). Portraits of artists: emergence of visual creativity in dementia. Archives of Neurology, 61(6), 842.

Montfort, N., and Fedorova, N. 2012. Small-scale systems and computational creativity. Third International Conference on Computational Creativity, 82-86.

Mora, E. (2006). Collective production of creativity in the Italian fashion system. Poetics, 34(6), 334-353.

Moran, S., and John-Steiner, V. 2003. Creativity in the making. In Sawyer, R. K., JohnSteiner, V., Moran, S., Sternberg, R. J., Feldman, D. H., Nakamura, J., and Csikszentmihalyi, M., eds., Creativity and Development, Oxford: Oxford University Press. 61-90.

Morin, E. (1992) Method, Towards a Study of Humankind. Peter Lang Publishing. Morris, R. G., Burton, S. H., Bodily, P. M., \& Ventura, D. (2012). Soup over bean of pure joy: Culinary ruminations of an artificial chef. Third International Conference on Computational Creativity, 119-125.

Nemeth, C. J., Personnaz, B., Personnaz, M., \& Goncalo, J. A. (2004). The liberating role of conflict in group creativity: A study in two countries. European Journal of Social Psychology, 34(4), 365-374.

Noori, M. M., Ahadi, H., Askari, P., Hashemian, K., \& Mazaheri, M. M. (2012). The link between creativity and mental health among the students of the universities in Tehran. Journal of American Science, 8(8).

Nowak, A., \& Lewenstein, M. (1996). Modelling social change with cellular automata. In Modelling and Simulation in the Social Sciences from the Philosophy of Science Point of View (pp. 249-285). Springer.

Ohly, S., Kase, R., \& Škerlavaj, M. (2010). Networks for generating and for validating ideas: The social side of creativity. Innovation: Management, Policy \& Practice, 12(1), 41-52.

Otte, A. (2001). The plasticity of the brain. European Journal of Nuclear Medicine and Molecular Imaging, 28(3), 263-265. 
Paulus, P. B., \& Nijstad, B. A. (Eds.). (2003). Group Creativity: Innovation through Collaboration. Oxford University Press.

Paulus, P. B., Levine, D. S., Brown, V., Minai, A. A., \& Doboli, S. (2010). Modelling ideational creativity in groups: connecting cognitive, neural, and computational approaches. Small Group Research, 41(6), 688-724.

Rosenthal, R. N., \& Westreich, L. M. (2010). Cosmetic psychopharmacology: drugs that enhance wellbeing, performance, and creativity. Clinical Addiction Psychiatry, 72.

Russell, S. J., and Norvig, P. (1995). Artificial Intelligence: A Modern Approach. Prentice Hall.

Sak, U. (2004). About creativity, giftedness, and teaching the creatively gifted in the classroom. Roeper Review, 26(4), 216-222.

Saracho, O. (2012). Creativity theories and related teachers’ beliefs. Early Child Development and Care, 182(1), 35-44.

Saunders, M. N., Saunders, M., Lewis, P., \& Thornhill, A. (2011). Research Methods for Business Students, Pearson Education.

Sawyer, R. K. (2006). Group creativity: Musical performance and collaboration. Psychology of Music, 34(2), 148-165.

Scott, A. J. (2006). Creative cities: conceptual issues and policy questions. Journal of Urban Affairs, 28(1), 1-17.

Shalley, C. E., \& Gilson, L. L. (2004). What leaders need to know: A review of social and contextual factors that can foster or hinder creativity. The Leadership Quarterly, 15(1), 33-53.

Sheldon, K. M., Cheng, C., \& Hilpert, J. (2011). Understanding well-being and optimal functioning: Applying the multilevel personality in context (MPIC) model. Psychological Inquiry, 22(1), 1-16.

Shepard, B. (2011). Play, Creativity, and Social Movements: If I Can't Dance, It’s Not My Revolution. Routledge.

Simonton, D. K. (2004). Creativity in Science: Chance, logic, genius, and zeitgeist. Cambridge University Press.

Smith, S. M., Gerkens, D. R., Shah, J. J., \& Vargas-Hernandez, N. (2006). Empirical studies of creative cognition in idea generation. Creativity and innovation in organizational teams, 3-20. 
Smith, S. M., Ward, T. B., \& Finke, R. A. (Eds.). (1995). The Creative Cognition Approach. MIT Press.

Snyder, A. W., Ellwood, S., \& Chi, R. P. (2012). Switching on creativity. Scientific American Mind, 23(5), 58-62.

Sosa, R. and Gero, J.S. (2012). Brainstorming in solitude and teams: the role of group influence, Third International Conference on Computational Creativity, 188196.

Sosa, R., \& Gero, J. S. (2005). A computational study of creativity in design: The role of society. Artificial Intelligence in Engineering, Design and Manufacturing (AIEDAM), 19(04), 229-244.

Sosa, R., \& Gero, J. S. (2013). Multilevel computational creativity. Fourth International Conference on Computational Creativity, pp. 198-204.

Sosa, R; Gero, J.S.; Jennings, K. (2009). Growing and destroying the worth of ideas, Proceedings of the 7th ACM Conference on Creativity and Cognition 2009, 295304.

Starko, A. J. (2009). Creativity in the Classroom: Schools of Curious Delight. Routledge.

Sternberg, R. J., \& Grigorenko, E. L. (2001). Unified psychology. The American Psychologist, 56(12), 1069-1079.

Storm, B. C., \& Angello, G. (2010). Overcoming Fixation. Psychological Science, 21(9), 1263-1265.

Sunstein, C. R. (2003). Why Societies Need Dissent. Harvard University Press.

Syed, M. (2010). Bounce: Mozart, Federer, Picasso, Beckham, and the Science of Success. Harper.

Tan, A. G. (1997). What can we learn from a century of cultivating creative thinking in Japan?. Teaching and Learning, 18(1), 9-18.

Tierney, P., \& Farmer, S. M. (2011). Creative self-efficacy development and creative performance over time. Journal of Applied Psychology, 96(2), 277.

Torrance, E. (1981). Predicting the creativity of elementary school children (1958-80) and the teacher who “made a difference.” Gifted Children Quarterly, 25 (2), 5562.

Van Mierlo, H., Rutte, C. G., Kompier, M. A., \& Doorewaard, H. A. (2005). SelfManaging Teamwork and Psychological Well-Being Review of a Multilevel Research Domain. Group \& Organization Management, 30(2), 211-235. 
Vygotsky, L. S. (1990). Imagination and creativity in childhood. Journal of Russian and East European Psychology, 28(1), 84-96.

Webb, G. R., \& Chevreau, F. R. (2006). Planning to improvise: the importance of creativity and flexibility in crisis response. International Journal of Emergency Management, 3(1), 66-72.

Weisberg, R. (1993). Creativity, Beyond the Myth of Genius, W.H. Freeman and Company, New York.

Westmeyer, H. 2009. Kreativität als relationales Konstrukt. In Witte, E.H., and Kahl, C.H., eds., Sozialpsychologie der Kreativität und Innovation, 11-26. Lengerich: Pabst Science Publishers.

Wong, T. (2012). To copy or not to copy, that is the question: the game theory approach to protecting fashion designs. University of Pennsylvania Law Review, 160, 1139.

Zhang, W., \& Niu, W. (2013). Creativity in the later life: Factors associated with the creativity of the Chinese elderly. The Journal of Creative Behavior, 47(1), 6076. 


\begin{tabular}{|l|l|}
\hline MDC scales & Sample creativity studies \\
\hline MDC-1: & $\begin{array}{l}\text { Cultural dimensions in creativity (Lubart 2010); Peer-reviewed repositories (Duflou and } \\
\text { Verhaegen 2011); IP law (Lessig 2008); Built environment (McCoy and Evans 2002). }\end{array}$ \\
\hline MDC-2: & $\begin{array}{l}\text { Gatekeeping (Sosa and Gero 2005); Cultural psychology (Glăveanu 2010); Creative } \\
\text { class (Florida 2005); Migration (Hansen and Niedomysl 2009); Social capital (Fischer } \\
\text { et al. 2004). }\end{array}$ \\
\hline MDC-3: & $\begin{array}{l}\text { Group conformity (Kaplan et al. 2009); Team diversity (Bassett-Jones 2005); Group } \\
\text { brainstorming (Sosa and Gero 2012). }\end{array}$ \\
\hline MDC-4: & $\begin{array}{l}\text { Creative cognition (Finke et al. 1992); Bilingüalism (Adesope et al. 2010); } \\
\text { Extraversion and dominance (Anderson and Kilduff 2009); Openness (Dollinger 2004); } \\
\text { Mndividual }\end{array}$ \\
\hline Brain & Functional neuroanatomy (Dietrich and Kanso 2010; Jung et al. 2010); Neural network \\
& models (Iyer et al. 2009)
\end{tabular}

Table 1. Sample studies of creativity in the five scales of our multi-dimensional model of creativity (MDC). 


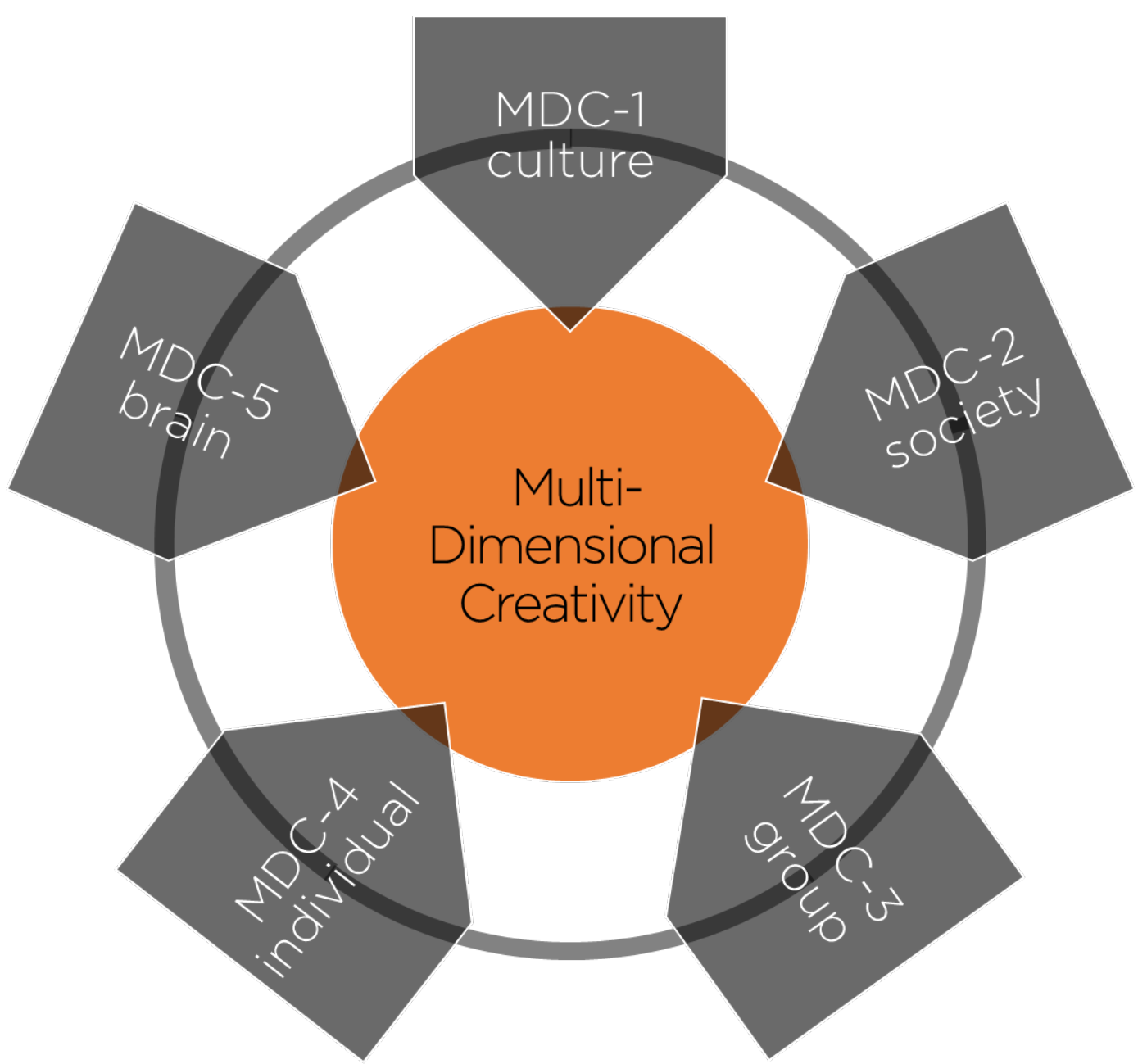

Figure 1. Radial arrangement of MDC scales show that rather than subsumption of lower levels, scale-specific factors exist that are not decomposable to smaller units. 


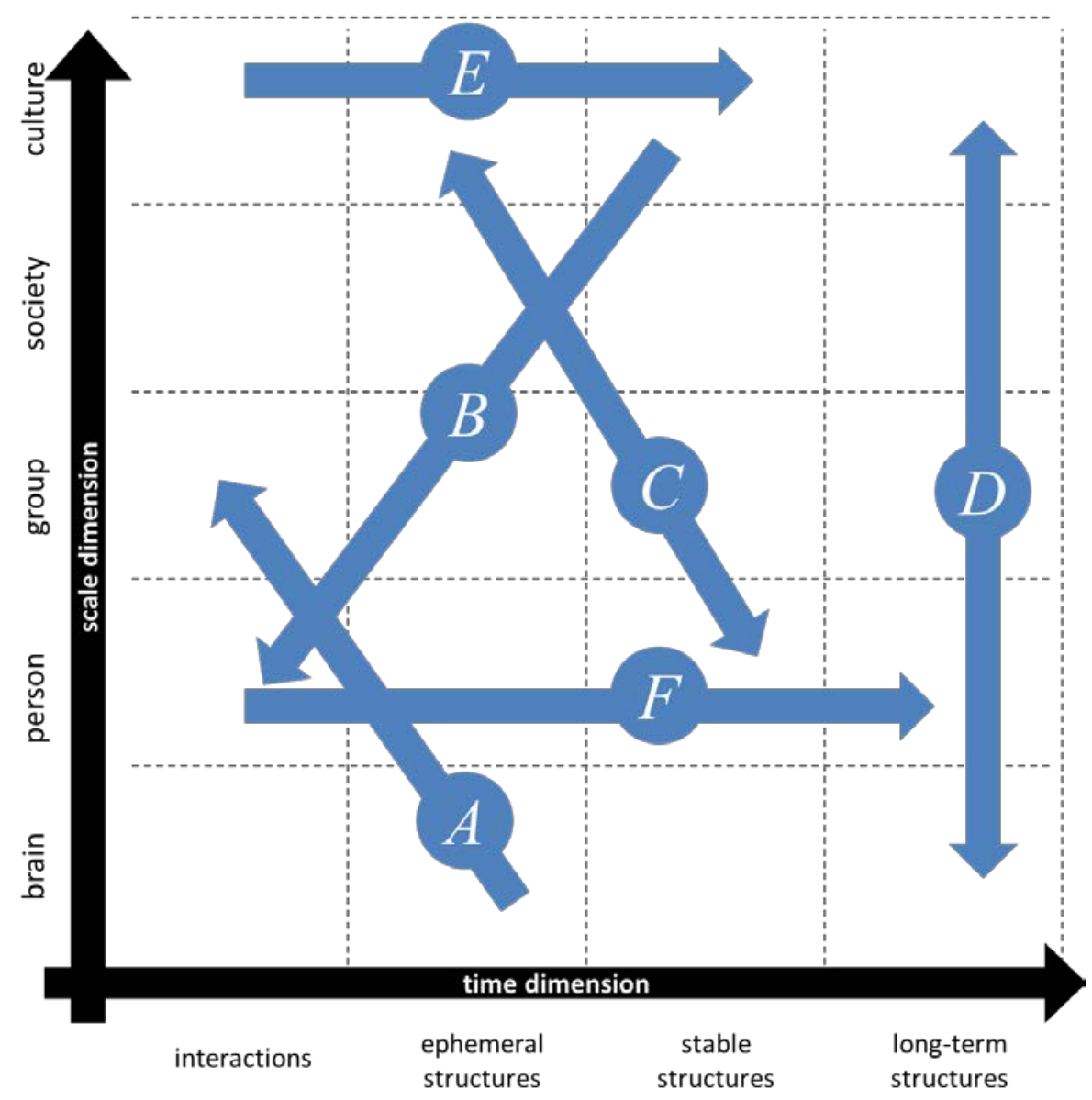

Figure 2. MDC dimensions (axes) and modeling approaches (arrows).

\begin{tabular}{|c|c|c|c|c|c|}
\hline Authors & $\begin{array}{l}\vec{U} \\
\stackrel{3}{\Sigma}\end{array}$ & $\begin{array}{l}\text { Ũ } \\
\text { ¿ }\end{array}$ & 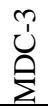 & 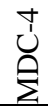 & 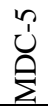 \\
\hline Agustini & • & & & • & \\
\hline Baydin & $\bullet$ & & & - & \\
\hline Burnett & $\bullet$ & $\bullet$ & & $\bullet$ & \\
\hline Charnley & & & & $\bullet$ & \\
\hline Colton & $\bullet$ & & & $\bullet$ & \\
\hline Eigenfeldt & & - & & $\bullet$ & \\
\hline Gabora & $\bullet$ & & & $\bullet$ & $\bullet$ \\
\hline Gatti & - & & & - & \\
\hline Grace & $\bullet$ & & & $\bullet$ & \\
\hline Hoover & $\bullet$ & & & $\bullet$ & $\bullet$ \\
\hline Indurkhya & $\bullet$ & - & & $\bullet$ & \\
\hline Jennings & & & & $\bullet$ & \\
\hline Johnson & - & - & & $\bullet$ & \\
\hline Jordanus & $\bullet$ & & & & \\
\hline Jursic & $\bullet$ & & & $\bullet$ & \\
\hline Keller & & & & $\bullet$ & \\
\hline $\mathrm{Li}$ & & & & $\bullet$ & \\
\hline Linson & & & $\bullet$ & $\bullet$ & \\
\hline Maher & - & - & $\bullet$ & & \\
\hline Monteith & $\bullet$ & & & $\bullet$ & \\
\hline Montfort & & & & $\bullet$ & \\
\hline Morris & - & & & $\bullet$ & \\
\hline Noy & & & & $\bullet$ & \\
\hline O’Donoghue & - & & & $\bullet$ & \\
\hline
\end{tabular}




\begin{tabular}{|l|l|l|l|l|l|} 
Ogawa & $\bullet$ & & & $\bullet$ & \\
\hline Pérez y Pérez & $\bullet$ & $\bullet$ & & $\bullet$ & \\
\hline Rank & & & & $\bullet$ & \\
\hline Ritchie & & & & $\bullet$ & \\
\hline Smith & $\bullet$ & & & $\bullet$ & \\
\hline Sosa & & & $\bullet$ & $\bullet$ & \\
\hline Toivanen & $\bullet$ & $\bullet$ & & $\bullet$ & \\
\hline Veale & $\bullet$ & & & $\bullet$ & \\
\hline Wiggins & & & & $\bullet$ & \\
\hline Zhu & $\bullet$ & & & & \\
\hline
\end{tabular}

Table 2. Classification of recent computational creativity papers using MDC levels

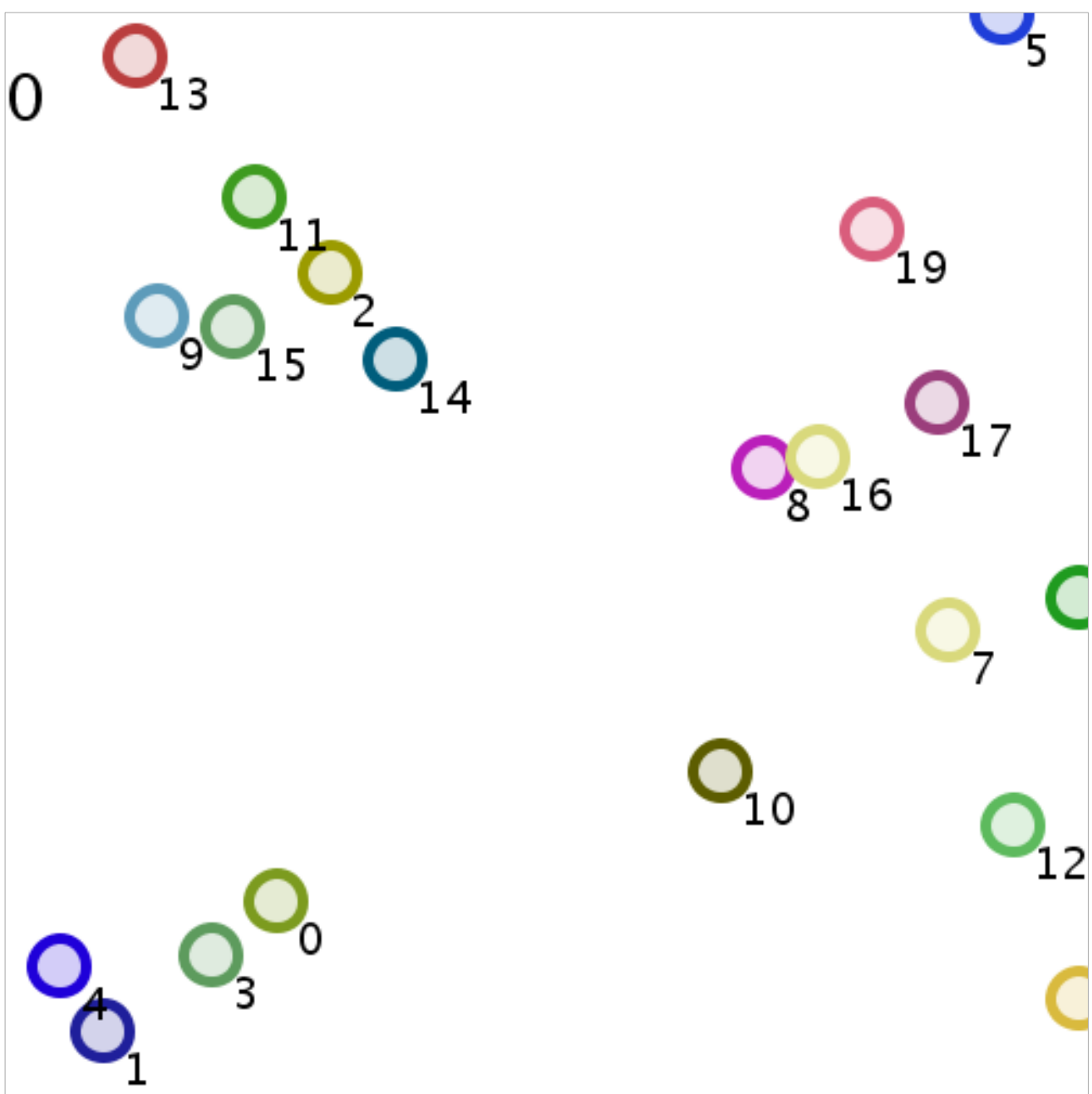

Figure 3. Typical initial state of a cellular automata of 20 mobile agents in a torus square grid of size 200 x 200. Color coding represents the 'idea values' assigned at random at initial step. 


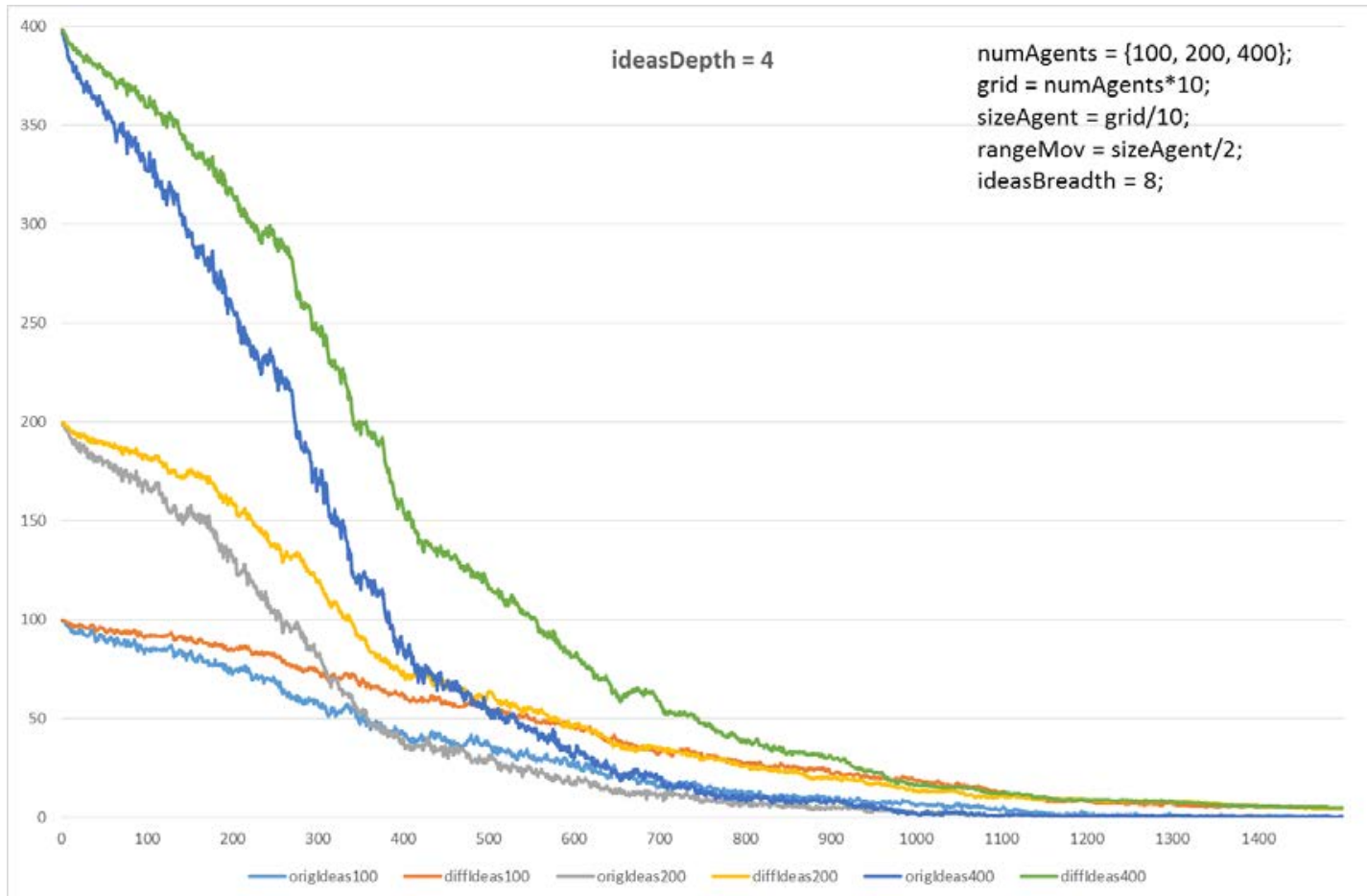

Figure 4. Set of results with 100, 200 and 400 agents showing total group convergence across cases.

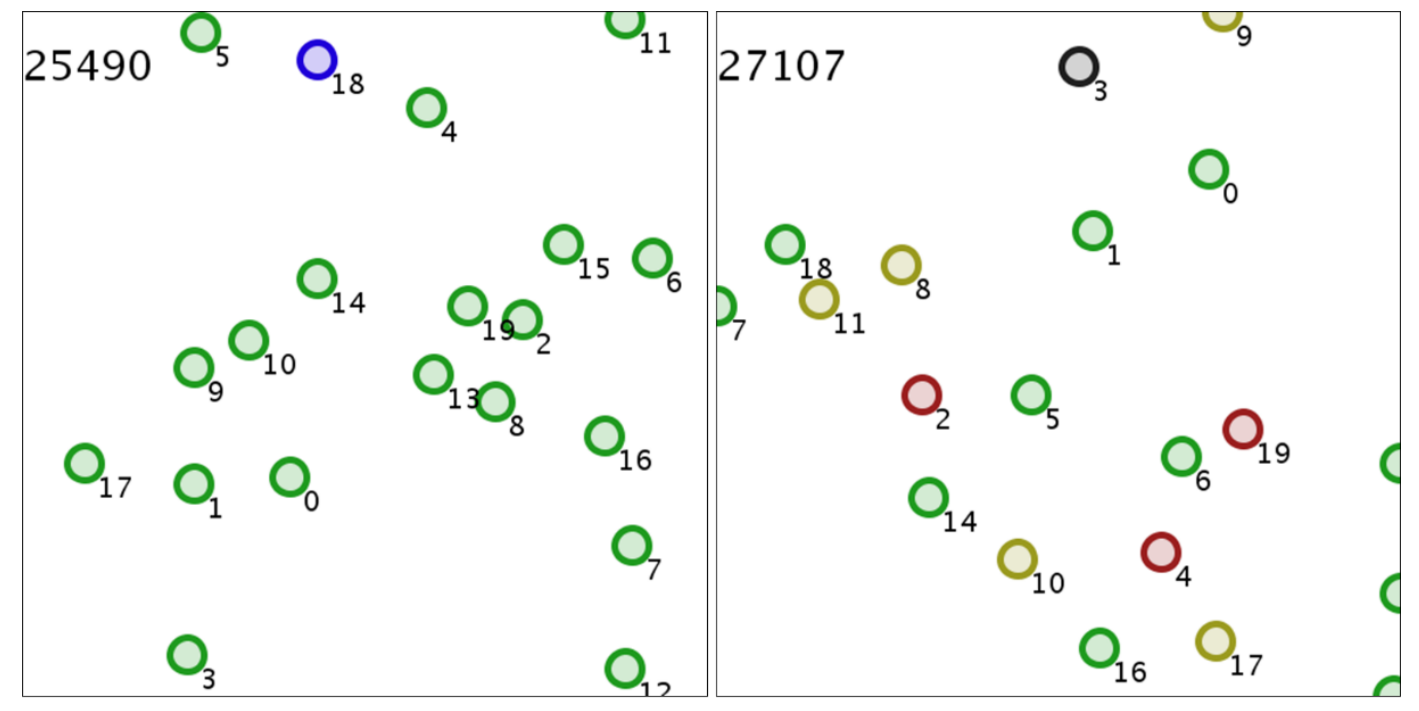

(a)

(b)

Figure 5. Generative process by one agent leading to the spread of new ideas in a group. 


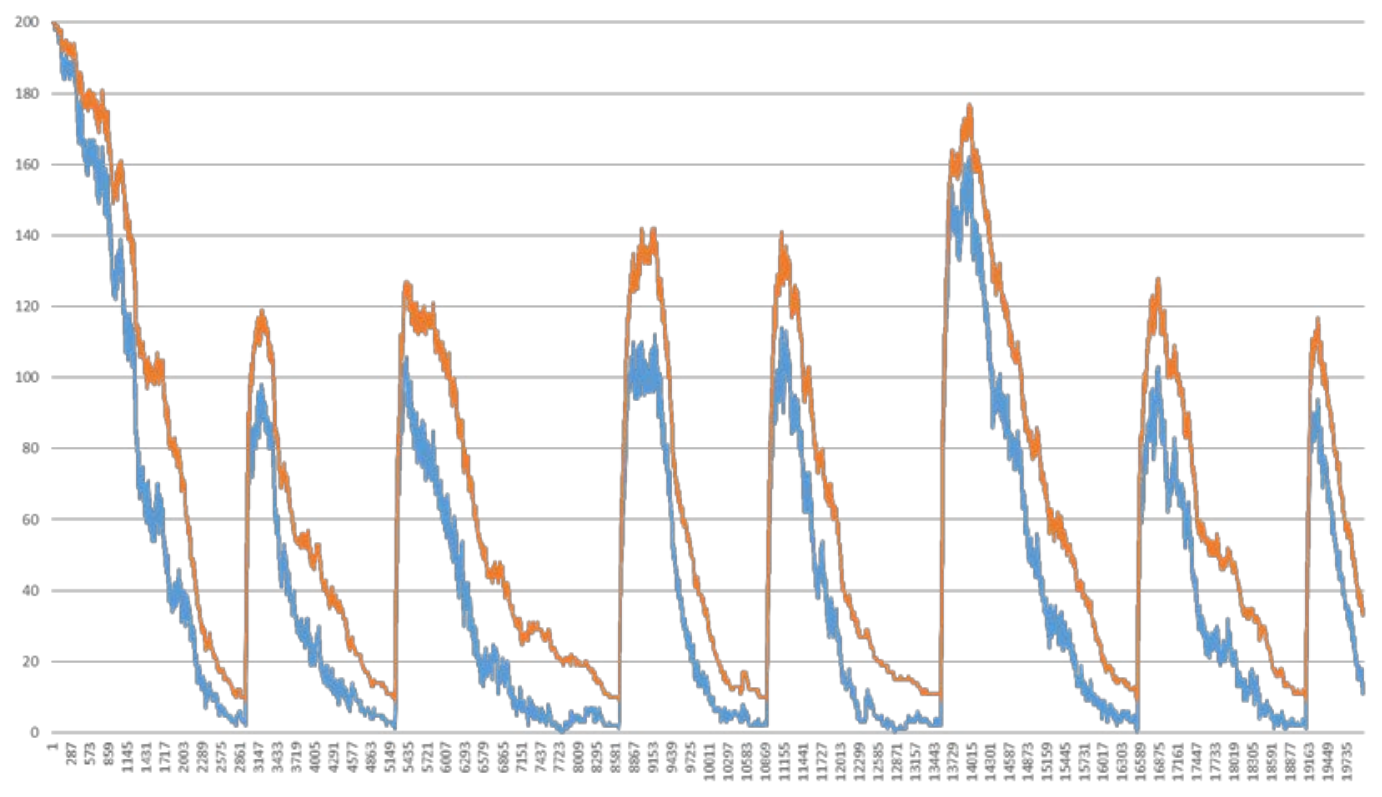

Figure 6. Diversity effects of non-conformist behavior: a group of 200 agents going through cycles of divergence and convergence. 